\title{
SIAR: an autonomous ground robot for sewer inspection
}

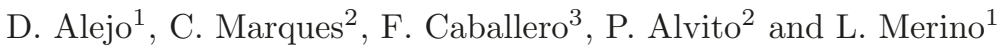 \\ ${ }^{1}$ School of Engineering, Universidad Pablo de Olavide, Crta. Utrera km 1, \\ Seville, Spain. Email: \{daletei,lmercab\}@upo.es \\ ${ }^{2}$ IDMind, Lisbon, Portugal. Email: \{cmarques,palvito\}@idmind.pt \\ ${ }^{3}$ Dpt. of System Engineering and Automation, University of Seville, \\ Camino de los Descubrimientos, s/n, Seville, Spain. Email: fcaballero@us.es
}

\section{Resumen}

This paper summarizes the latest advances of the EU Project SIAR-ECHORD++, whose main objective is the design of a new robotic platform for inspecting visitable sewers. The SIAR robot aims to determine the sewer serviceability, to identify critical structural defects, to perform sewer monitoring and eventually to take water or gas samples of the environment. To this end, an autonomous $I P 67$ ground robot equipped with $R G B-D$ sensors and with a powerful wireless communication system is being developed. It is able to perform $3 D$ structure reconstruction in real-time that could be used to detect defects in the sewer structures. This paper presents the key aspects in the design of the platform as well as preliminary experimental results in real sewer systems.

Keywords: Autonomous ground robot, sewer inspection, 3D reconstruction, sensing, navigation

\section{Introduction}

Sewer inspections require many people to work in risky and unhealthy conditions. Moreover, sewers are classified as confined spaces which require special health and safety measures, in addition to other risks present like slippery sections, obstacles or biological risks from the potential contact with wastewater. These features make the process of sewer inspection a risky and expensive process that requires improvements urgently. Therefore, introducing a robotic solution in this process aims at reducing the labour risks, improving the precision of sewer inspections and optimizing sewer cleaning resources of the city.

Autonomous sewer inspection by robots is a harsh task in many senses: the environment is very aggressive with a high level of humidity and potential toxic gases; the robot should move in very narrow spaces with almost no place for maneuvering; and communications are very restricted due to lack of direct line of sight (NLOS). In addition, the robot needs to carry a wide range of sensors not only for self-localization and navigation, but also to perform the inspections tasks: visual imaging with artificial illumination, 3D scanning sensors for metric environment reconstruction to facilitate impairment detection or devices for air/waste sampling. These sensors increase the weight of the system and also drain the power of the robot with the corresponding impact in battery life and autonomy.

Payload, autonomy (in terms of battery life and traversed distance) and harsh environment altogether conform a working framework in which ground robots seem to be the best solution [13]. Additional options include the use of micro aerial vehicles (MAVs) based on multi-rotors, given their small size and maneuverability. However, there are limitations that need to be considered, as the limited payload capacity that current systems offer, in order to carry the required sensors without compromising the robot autonomy; even tethered multi-rotors might not have enough payload due to the additional weight added by the power cable. In addition, to the best of our knowledge there are no water resistant commercial MAVs in the market, a key factor for a robust sewer inspection robot. On the other hand, submarine robots are discarded because the water level into the sewage network is expected to be low during inspection and monitoring tasks and the inspection capabilities from inside the water are very limited. Thus, ground robots are the best choice if long-term and high maneuverability are required, as they can be built waterproof and sensors of different size and weight can be installed onboard.

There are a wide variety of robotic platforms for gallery inspection already in the market. In particular, the sewer inspection scene can be divided in two fields: pipe inspection performed by small robots which can fit inside pipes; and sewer inspection performed by medium to big sized robots which move inside galleries. Pipe inspection can be purely remote controlled, e.g., Alligator, Minigator, Multigator and Flexigator wheeled robots from IBAK [2] or Geolyn's tracked robots [1], but it can be also autonomous, e.g., Solo tracked robots from REDZone [6] or Makro's wheeled worm type robot [3]. In sewer inspection 
is also possible to find teleoperated solutions, e.g., PureRobotics' Pipeline Inspection tracked robot [4], and autonomous solutions, e.g., ServiceRoboter wheeled solution from Fraunhofer IFF [5]. However, they have been designed in order to inspect small pipes and sewers and cannot be used inside the visitable sewers.

The SIAR (Sewer Inspection Autonomous Robot) solution seeks to solve the problem by the creation of an autonomous robot, with the possibility of control from a human operator in case of need or emergency. By using the proposed wireless system, augmented by the deployment of selfpowered wireless repeaters, it will be possible to transmit bidirectional data between robot and an external operator, allowing the operator to change the mission in real time, instead of having to wait for the end of the sewer exploration to identify problems on the sewer system. The SIAR system will go beyond existing solutions through the inclusion of some innovative features, while maintaining affordable costs: configurable locomotion system with interchangeable wheels and tracks modules and variable length/width of the traction system; reliable navigation system based on data fusion from low-cost commercial RGB-D cameras, IMU and encoders, performing accurate localization and navigation on the sewer system, instead of using more complex and expensive sensors for the same task; inspection system with easy exchangeable sensor payload, allowing the use of the robot in different applications instead of the usually set of sensors used only for sewer inspection, and the introduction of devices for air/waste sampling that is not possible to find in the commercial inspection robots.

The rest of the paper is structured as follows. First Section 2 describes the main hardware parts of the SIAR platform. Then, Section 3 describes the main parts of the system architecture integrated in SIAR. Section 4 describes the results obtained in initial experiments performed in real scenarios. Finally, Section 5 points out the conclusions obtained from the early results and the challenges that are still remaining.

\section{SIAR platform}

The SIAR project will develop a fully autonomous ground robot able to navigate through the sewage system with a minimal human intervention and with the possibility of manually controlling the vehicle or the sensor payload when required. In this section, the main components of the platform are described and discussed.

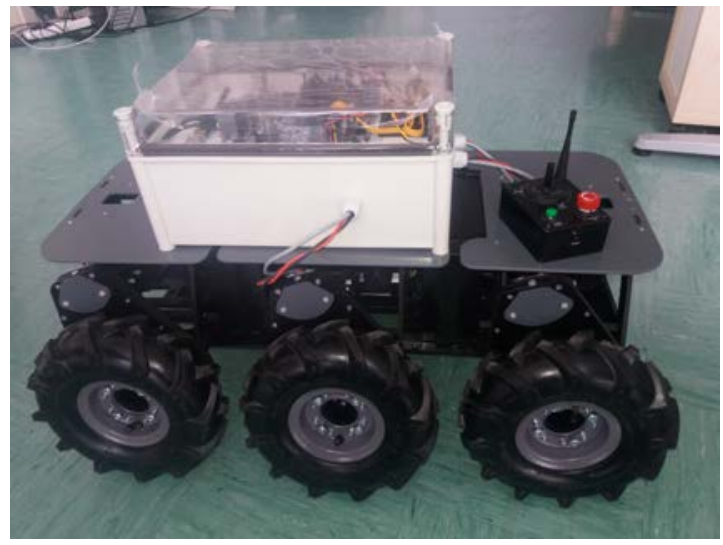

Figure 1: SIAR robotic platform

\subsection{Robot frame}

Figure 1 presents the current robotic platform used in real experimental scenarios. The robotic frame is made with IP67 enclosings in order to accommodate for the hardest environmental conditions during sewer inspection. A six-wheeled ground robot with independent traction has been designed and implemented by the company IDMind as part of this work. It is able to navigate over a wide range of floors and small obstacles, including steps of over $20 \mathrm{~cm}$. The axis of the two central wheels is positioned below the other ones, and the centre of gravity of the robot is located over that axis, so that for turning the robot can behave closely to a differential-drive robot. This configuration is very flexible and adapts very well to the different scenarios the robot can find during routine inspections. While this is not the final solution for the problem, it allows to test the locomotion concept. It carries LiFePo batteries for batteries and electronics, with an autonomy of 5 hours.

\section{$2.2 \quad$ Sensing systems}

There are several kind of sensors that can provide a robot with detailed measurements of the environment. These sensors include 2D LiDAR such as Hokuyo, 2D tilting laser that can obtain 3D measures, and 3D LiDAR sensors such as the velodyne HDL-32E [7], stereo cameras and RGB$\mathrm{D}$ cameras.

When analyzing these options, 2D laser can obtain precise measures at tens of Hertzs, but only can sense distances confined in a determinate plane local to the robot. Therefore, it can be used to sense obstacles in the surroundings, but it would have to be complemented if holes or obstacles on the floor have to be detected, or in order to perform a $3 \mathrm{D}$ scan of the environment. On the other hand, 


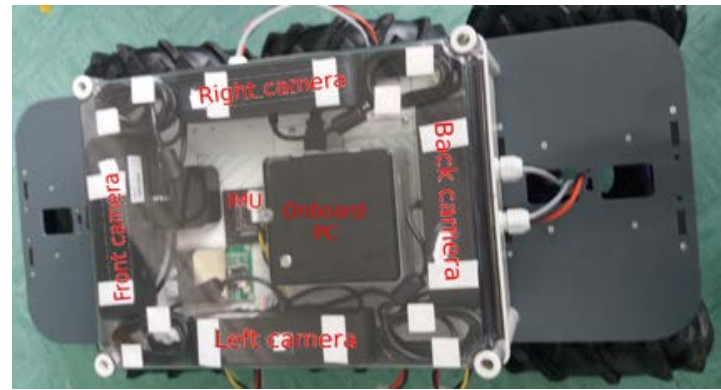

Figure 2: Detail of the sensors and computing unit in the SIAR platform

tilted laser sensors could be employed to perform hole detection and 3D scans, but they offer the data at a medium rate (Hertzs). 3D LiDAR sensors can provide the robot with a detailed $3 \mathrm{D}$ data of its surrounding usually at tens of Hertzs, but they are very expensive (thousands of dollars) and heavy (usually more than one kilogram). Stereo cameras would need a good illumination in order to be able to detect matches between the two cameras, and then compute their disparity. This is not the case when inspecting sewers.

Therefore, RGB-D cameras emerge as a very convenient alternative for performing $3 \mathrm{D}$ scans of the surroundings of the robot as they offer distance measures at high frequencies $(30 \mathrm{~Hz})$ even without the presence of illumination. Also they are inexpensive (around 150 Euro) when compared to the other Radar and LiDAR alternatives.

\subsubsection{Current solution}

The current SIAR platform counts with 4 RGBD cameras from Orbbec $^{1}$ disposed in the front, rear, left and right parts of it as depicted in Figure 2. The front and rear sensors are special RGB-D cameras with extended range that make them suitable for navigation purposes. Additionally, two lateral RGB-D cameras (left and right) are used for obtaining more precise measures of the structureś walls.

\subsubsection{Proposed design}

In the proposed design, the number of sensors will increase to 5 sensors (see Figure 3 ). In the front and rear parts there will be located two longrange RGB-D cameras (up to 8 meters) that will be mainly used for navigation and obstacle avoidance, although they also will be used for 3D reconstruction of the ground and the walls. The remaining three sensors have been placed in order to enhance the detection of possible structural defects at the ceiling and in the upper parts of the

\footnotetext{
${ }^{1}$ https://orbbec3d.com
}

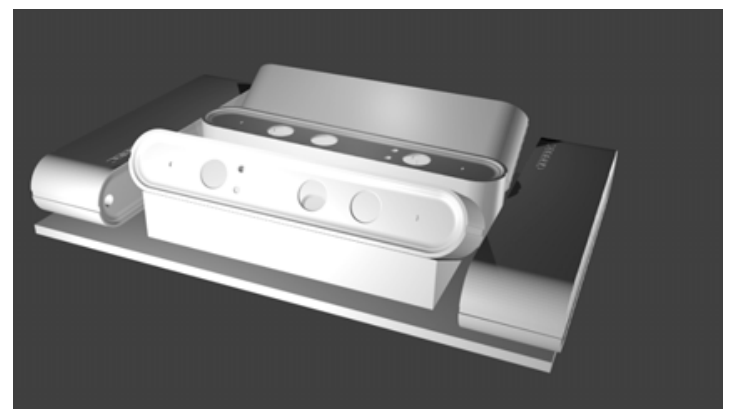

Figure 3: Proposed disposition of the cameras in the SIAR platform

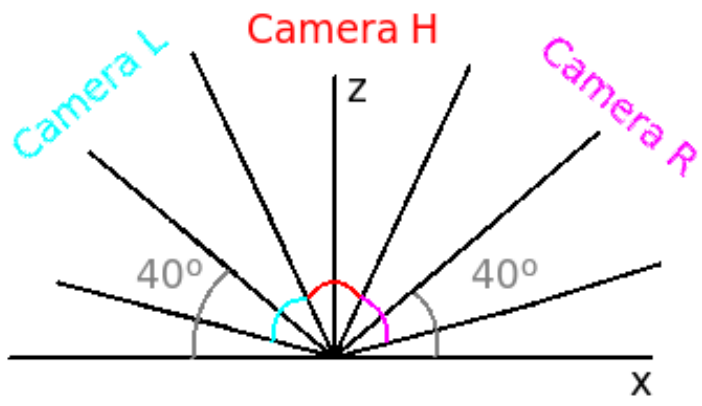

Figure 4: Detail of the angular disposition of cenital and lateral cameras for obtaining a continuous point cloud. Cenital camera (Camera $\mathrm{H}$ ) is pointing towards the ceiling, while lateral cameras are rotated 40 degrees in the $\mathrm{x}$ axis

walls. The vertical disposition of the last three sensors is detailed in Figure 4. Note that the the FOV of the sensors has taken into account in order to obtain a continuous point cloud of the environment. The sensors will be enclosed with IP67 protection as well. A specific computer will be devoted to the capture and managing of the data from the 5 cameras.

\subsection{Communication systems}

The design of a wireless communication system inside the sewer system involves a great challenge due to the harsh environmental conditions. In fact, it is common to find $100 \%$ humidity condition. Another major issue is the presence of obstacles, such as walls, pipes, garbage which would not allow LOS transmission between the robot and the base. In these circumstances, it is well known that signals with lower frequency would have better penetration properties than the ones with higher frequency.

In the proposed solution, the robot will be able to communicate with the base station by two independent data links as follows.

1. Primary link. The robot will be equipped 
with a long range $\mathrm{WiFi}$ in order to provide the computers onboard of the robot and the comms package with $\mathrm{WiFi}$ connectivity ${ }^{2}$. They have an advertised range of $50 \mathrm{~km}$, that will likely drop to less than $1 \mathrm{~km}$ in indoors environments. This link will provide the robot with a high bandwidth connection (up to $54 \mathrm{Mbps}$ ) that could be used for video streaming and sending additional data information such as the generated 3D map of the environment, localization to name a few.

2. Backup link. The robot and base station will also be equipped with Microhard $\mathrm{P} 900^{3}$ equipments. This link is able to connect two devices separated up to $50-100 \mathrm{~km}$ with a data rate of $236 \mathrm{Kbps}$ in outdoor environments with LoS. However, a reduction to the range of less than $1 \mathrm{~km}$ is expected in indoors. This range could be extended by the use of a directional antenna in one of the sides. This link will be used for reliable image transmission at low rates and reliable command transmission for teleoperation purposes.

Moreover, communication without direct LOS will be allowed by automatically deploying repeaters whenever the signal quality of the link goes below a predefined threshold level. In order to do that, the robotic platform will be augmented with a device to deploy and recover such repeaters. The alternative solution is the use of a tether, including power and communications, connected to the robot at all times. This scheme is employed by many robotic systems for pipe inspection, but it limits greatly the maneuverability, and thus the ability of the robot to inspect the sewer. The use of multi-hop ad-hoc networks have been shown $[12]$ as an potential solution in similar scenarios.

\section{System architecture}

The systems that control the behavior of the SIAR robot have been developed with the Robotic Operating System ${ }^{4}(\mathrm{ROS})$ using the ros-indigo distribution under Ubuntu 14.04. Figure 5 depicts the architecture of the main modules present in the system:

1. IMU driver. This package filter the raw data produced by the inexpensive IMU sensor Arduimu v $3^{5}$.

\footnotetext{
${ }^{2}$ http://www.microhardcorp.com/nVIP2400.php

${ }^{3}$ http://www.microhardcorp.com/P900.php

${ }^{4}$ http://ros.org

${ }^{5}$ https://code.google.com/archive/p/ardu-imu/
}

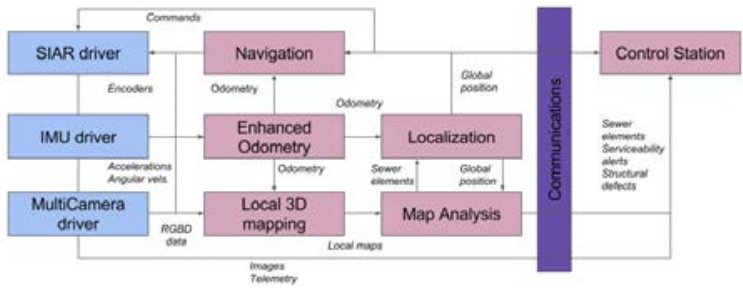

Figure 5: Software architecture

2. MultiCamera driver. This package extends the functionality of openni2_camera ${ }^{6}$ and openni2_launch ${ }^{7}$ packages. It provides the user with a utility for generating launch files that sequentially activate each camera. Each camera can be configured with its own setup, i.e. changing the image rate, its resolution and many more.

3. Enhanced odometry. This package provides enhanced odometry estimates by fusing IMU and encoders measures with visual odometry estimations [11]. The use of visually enhanced odometry is very relevant in these scenarios, where slippage is common. In this way, the SIAR platform is able to precisely and robustly estimate the relative displacements. A good odometry estimation is necessary for obtaining precise $3 \mathrm{D}$ maps of the environment[8].

4. Localization. These precise odometry estimations are used by this module in order to estimate the global position of the platform. This block uses prior information of the sewer network (localization of manholes, inlets, to name a few, available from a GIS) in order to correct the drift which is accumulated in the odometry estimation.

5. Local 3d mapping. This package gets the enhanced odometry and the precise localization outputs and uses them to integrate the measurements from the different RGB-D sensors disposed over the SIAR robot. This would allow the operator to have a precise $3 \mathrm{D}$ local or global reconstructions of the sewer.

6. Navigation. The SIAR robot will be commanded in three different operating modes. First, it can be purely teleoperated from the base station. Then, an assisted teleoperation method will be developed in order to make the robot automatically navigate through the center of the sewer, avoiding falling into holes,

\footnotetext{
${ }^{6}$ http://wiki.ros.org/openni2_camera

${ }^{7}$ http://wiki.ros.org/openni2_launch
} 
etc, while the operator command the velocity of the inspection. To this end, this module has to get a partial 3D reconstruction of the environment in order to detect the safe navigation areas where the robot can go through. Finally, a fully autonomous operating mode will use optimal kinodynamic sampling-based planners such as [10][9] to evaluate blocking configurations. This operating mode will further reduce the operator's workload, as complete inspection plans could be loaded to the platform, that will execute it automatically. Moreover, it would be crucial in case where the communication was lost. In this way, the platform can safely perform an automatic return home procedure.

7. Map analysis. This module provides the high level information required for sewer inspection. It receives the local $3 \mathrm{D}$ maps and analyzes them to extract sewer elements, estimate the serviceability and inspect critical defects. Furthermore, the sewer network elements like manholes and inlets, will be used by the localization module for global localization.

8. SIAR driver. Software that interfaces with the hardware board of the robot, and offers a standard ROS commands and services for easy integration of the robot with the rest of the system. This module will allow the operator to command actions to the SIAR which are not directly included in the navigation package, such as taking air or water measures and deploying repeaters.

9. Control Station. This module will send an alert to the operator when critical conditions occur. These conditions can include automatic detection of structural defects, bad state of the radio links, low battery indicator, the robot has lost traction and should be recovered, among others. It will also display the localization of the robot and the images from the onboard cameras in real-time.

\section{Experiments}

In this section, initial experimental results are described. In these experiments, the robot, in its current state, was deployed in part of the sewer system in Barcelona. The experiments were carried out in consecutive days on 2016, June 7-8. Each experiment lasted for three hours approximately. These initial were aimed at checking the basic capabilities of the platform. In the first day, a mobility test was carried out. The second day included a teleoperation test and an autonomy test.

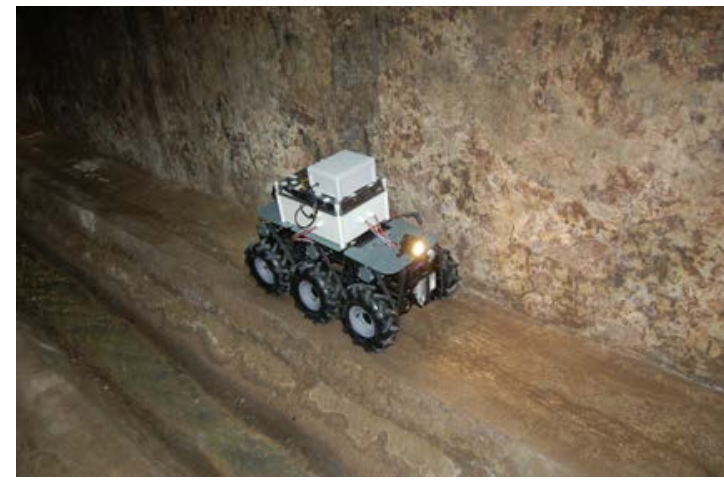

Figure 6: SIAR robot navigating in the sewers of the Passeig of Sant Joan

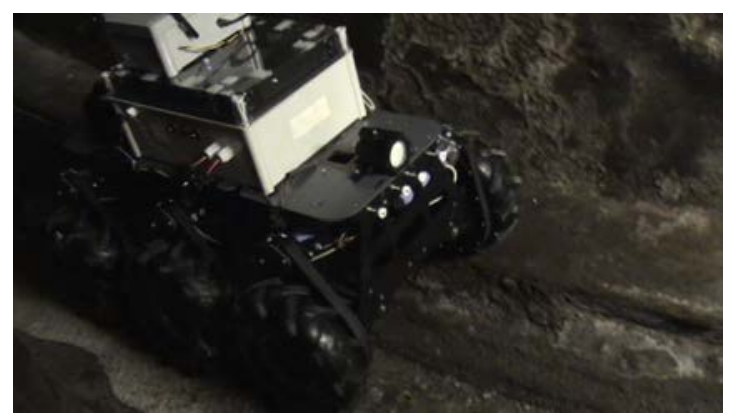

Figure 7: SIAR robot navigating in the sewers of Valencia street

\subsection{Scenario}

The particular location of the experiment consists of the sewers in Passeig Sant Joan, Barcelona (see Figure 6). This first part of scenario is a special part of the sewer which is open to guided visits. Therefore, it is wider than usual and is provided with an illumination system. However, the SIAR platform has also to be designed to travel inside regular sewers such as the depicted in Figure 7. These types of sewers are much narrower, are not illuminated, and present noticeable irregularities in their structure, which make the navigation in such scenarios challenging.

Figure 8 depicts the sections of the different types of sewers that have to be visited by the platform. The first section corresponds with the Passeig of Sant Joan. Then, the other sections are the most common sections present in the regular sewers. Note that the width of the SIAR platform had to be carefully tuned so the platform can navigate in the sides of the sewers of the Passeig of Sant Joan, while being also capable of navigating over the gully (one wheel at each side) of the other sewers. A map with the total travel to be carried out can be found in Figure 9 . 


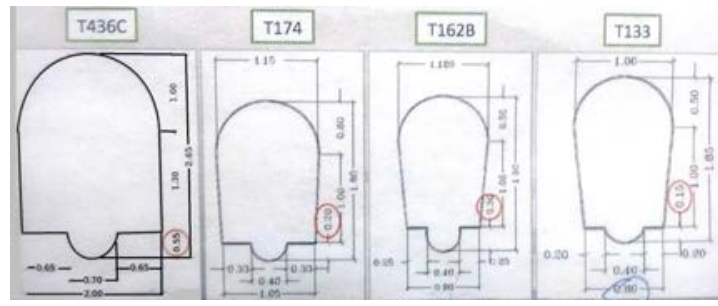

Figure 8: Sections of the sewers to be visited by the SIAR platform

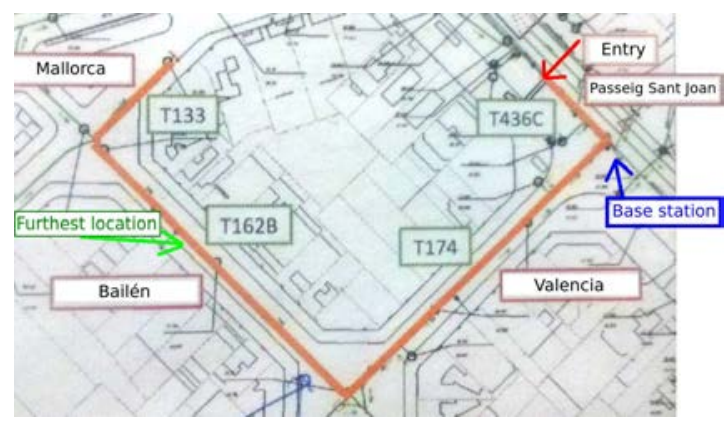

Figure 9: Total travel carried out by the SIAR platform

\subsection{Mobility and autonomy results}

The platform has been able to continuously operate for 6 hours without battery recharges in the considered scenario, with a total traveled distance of almost $2 \mathrm{~km}$.

In each experiment the start location was the entry of the sewers, marked by a red arrow in Figure 9. The first mission was a mobility test in which the SIAR platform was directly teleoperated behind the robot at a close distance. In this experiment, the robot was able to navigate to the furthest location marked in a green arrow.

In the autonomy test, the robot was commanded to travel the sewer at the Passeig of Sant Joan for a one-way distance that exceeded $200 \mathrm{~m}$. The SIAR platform was able to perform more than four travels, thus covering almost $1 \mathrm{~km}$ of total traveled distance in this test. The test was carried out partly teleoperated from the base station (one of the ways) and the rest of the time was directly operated.

\subsection{Communication and teleoperation results}

One of the main objectives of the experiment was to check the communication systems between the base station and the robot. In the mobility test, the base station received a video link of the RGB$\mathrm{D}$ cameras without interruptions with low delays (less than 0.2s). Communication without LOS

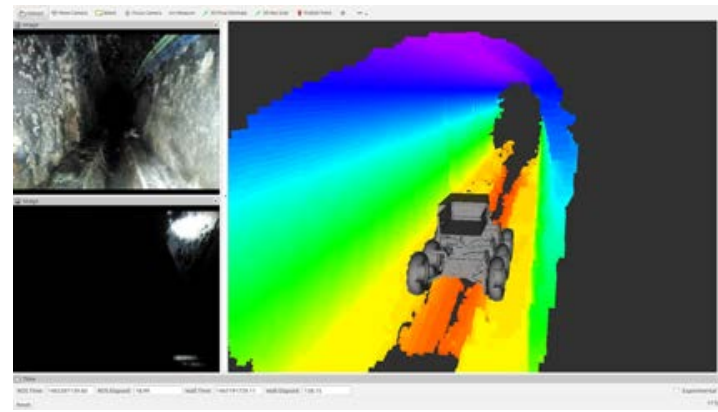

Figure 10: 3D local map reconstruction using RGBD data.

was achieved thanks to the manual deployment of a repeater just in the corner of the travel, this location is marked in purple in Figure 9.

Moreover, this video link was used in the experiments of the following day in order to teleoperate the robot from the base station. In the teleoperation experiments, the robot reached a distance of roughly $50 \mathrm{~m}$ without any assistance. Then, the back-up link in which the commands were sent was broken and therefore the robot was directly operated back to the Base Station. This bad performance of the $900 \mathrm{MHz}$ had not been detected in experiments outside the sewer, in which its coverage was greater than the $2.4 \mathrm{WiFi}$ link.

Finally, the platform was teleoperated in one of the ways in the autonomy test without experiencing any difficulties or communication issues.

\subsection{D reconstruction results}

The systems described in Section 3 have been used to produce a detailed $3 \mathrm{D}$ reconstruction of the sewer system in real-time. Figure 10 represents a snapshot of the obtained 3D model along with the actual images that were get from the front camera. Note that the resolution of the reconstruction could allow the operator to easily detect imperfections in the structure of the sewer.

\section{Conclusions}

The paper has discussed the main design decisions for the development of a sewer inspection robot for big city sewers. The paper describes briefly an initial platform used for tests, and the communication and sensing systems. Cost-effectiveness is one of the main issues to be considered. The paper also depicts the architecture of the system.

Furthermore, the paper presents preliminar results obtained in real sewers in tests related to autonomy, communications and mapping. These experiments illustrate that the decisions made go 
in the good direction.

As future work, the final design of the robotic platform and sensor payload will be pursued. New functionalities will be developed and tested in the next phases, including assisted teleoperation and navigation autonomy modes, and enhanced perception capabilities to support the monitoring of the sewers.

\section{Agradecimientos}

This work has been developed under the European project Echord++: European Coordination Hub for Robotics Development++ (FP7-601116) inside the challenge "Utility infrastructures and condition monitoring for sewer network. robots for the inspection and the clearance of the sewer network in cities".

\section{References}

[1] Geolyn. http://www.geolyn.ca/.

[2] Ibak. http://www.ibak.de/.

[3] Makro's wheeled worm robot. http: //www. inspector-systems.com/makro_ plus.html.

[4] Purerobotics' pipeline inspection. http: //www . puretechltd.com/services/ robotics/.

[5] Serviceroboter. http://www.iff. fraunhofer.de/de/geschaeftsbereiche/ robotersysteme/forschung/ serviceroboter-inspektion-reinigungwartung.html.

[6] Solo tracked robots from redzone. http://www.redzone.com/products/solorobots/.

[7] velodyne hdl-32e. http://velodynelidar. $\mathrm{com} / \mathrm{hdl}-32 \mathrm{e} . \mathrm{html}$.

[8] Huang, A. S., Bachrach, A., Henry, P., Krainin, M., Maturana, D., Fox, D., AND Roy, N. Visual odometry and mapping for autonomous flight using an rgb-d camera. International Symposium on Robotics Research (ISRR) 2 (2011).

[9] Jaillet, L., Cortés, J., And Simeon, T. Transition-based rrt for path planning in continuous cost spaces. In IEEE/RSJ International Conference on Intelligent Robots and Systems, IROS (2008).

[10] Karaman, S., and Frazzoli, E. Optimal kinodynamic motion planning using incremental sampling-based methods. In IEEE Conf. on Decision and Control (2010).
[11] Perez-Grau, F. J., Fabresse, F. R., CABallero, F., Viguria, A., AND Ollero, A. Long-term aerial robot localization based on visual odometry and radio-based ranging. In Proceedings of the 2016 International Conference on Unmanned Aerial Systems (Arlintong, USA, 2016).

[12] TARDioli, D. A proof-of-concept application of multi-hop robot teleoperation with online map building. In Proceedings of the 9th IEEE International Symposium on Industrial Embedded Systems (SIES 2014) (2014), IEEE, pp. 210-217.

[13] Tur, J. M. M., And Garthwaite, W. Robotic devices for water main in-pipe inspection: A survey. Journal of Field Robotics (2010), 491-508. 